\title{
New Thoughts on Thought Disorders in Parkinson's Disease: Review of Current Research Strategies and Challenges
}

\author{
Jennifer G. Goldman \\ Section of Parkinson Disease and Movement Disorders, Department of Neurological Sciences, Rush University Medical Center, \\ 1725 W. Harrison Street, Suite 755, Chicago, IL 60612, USA \\ Correspondence should be addressed to Jennifer G. Goldman, jennifer_g_goldman@rush.edu \\ Received 15 October 2010; Accepted 12 January 2011 \\ Academic Editor: Irena Rektorova \\ Copyright ( 92011 Jennifer G. Goldman. This is an open access article distributed under the Creative Commons Attribution \\ License, which permits unrestricted use, distribution, and reproduction in any medium, provided the original work is properly \\ cited. \\ Psychosis is a frequent nonmotor complication in Parkinson's disease (PD), characterized by a broad phenomenology and likely \\ due to a variety of intrinsic (i.e., PD-related) and extrinsic factors. Safe and effective therapies are greatly needed as PD psychosis \\ contributes significantly to morbidity, mortality, nursing home placement, and quality of life. Novel research strategies focused \\ on understanding the pharmacology and pathophysiology of PD psychosis, utilizing translational research including animal \\ models, genetics, and neuroimaging, and even looking beyond the dopamine system may further therapeutic advances. This review \\ discusses new research strategies regarding the neurobiology and treatment of PD psychosis and several associated challenges.
}

\section{Introduction}

Psychosis is a frequent nonmotor complication of Parkinson's disease (PD) [1-3]. Psychotic symptoms in PD manifest predominantly as hallucinations and delusions, although recently revised criteria for PD psychosis extend the spectrum to include illusions, a false sense of presence, hallucinations, and delusions [4]. These neuropsychiatric phenomena likely stem from a combination of drug-related (i.e., exogenous or extrinsic) factors and PD-related (i.e., endogenous or intrinsic) complications, although the exact pathophysiology of PD psychosis is unknown. Improved treatments for PD psychosis are greatly needed since hallucinations and delusions are important contributors to morbidity, mortality, nursing home placement, and quality of life [5-7]. To date, many clinical research trials for antipsychotic medications in PD have been affected by issues of trial design, medication side effects, and negative outcomes. As such, the goal of optimally designed clinical trials with effective and safe medications presents a challenge. Use of animal models or biomarkers (e.g., genetic or neuroimaging) may provide additional and complementary ways to advance treatments for PD psychosis. Thus, the aim of this review is to discuss new research strategies regarding $\mathrm{PD}$ psychosis and their associated challenges. This review will highlight research on nondopaminergic substrates, comorbid neuropsychiatric features often associated with PD psychosis and the potential roles for integrating in vivo neuroimaging, genetic risk factors, and animal models in studying PD psychosis, and lastly discuss challenges of medication trials.

Dopaminergic medications have been well recognized to induce psychosis in PD by stimulating or inducing hypersensitivity of mesocorticolimbic dopamine receptors [8-10]. Virtually all classes of anti-parkinsonian medications may produce psychosis in PD. Some studies suggest particular susceptibility with dopamine agonists compared to levodopa [11-15] and with anticholinergics especially in elderly PD patients [16]. While dopaminergic medications contribute to $\mathrm{PD}$ psychosis, exploration of other extrinsic and intrinsic factors is needed to advance our understanding of and our treatments for PD psychosis. Intrinsic or PD-related factors that may contribute to PD psychosis include abnormalities in the visual system; levels of visual dysfunction range from ocular pathology and retinal dopamine loss, impaired visual acuity and color or contrast discrimination, to disturbed attentional and visuospatial processing and abnormal cortical activation patterns [17-22]. Other factors, often noted in epidemiological studies, encompass clinical 
risk factors such as age, PD duration and severity, depression, cognitive impairment and dementia, and sleep disturbances $[2,3]$. PD hallucinators exhibit worse cognitive function overall including executive, attentional, and visuospatial abilities [23-26] and also have been observed to have greater sleep disturbances and REM behavior disorder [27-33]. Neuroimaging studies also suggest that there are distinct structural, functional, and metabolic abnormalities in PD hallucinators [20, 22, 34-40]. This review highlights several of these risk factors or comorbid neuropsychiatric features associated with $\mathrm{PD}$ psychosis as potential avenues for selective therapeutic strategies.

\section{Beyond Dopamine: Other Neurochemical Substrates and Neuropsychiatric Features Associated with PD Psychosis}

While nondopaminergic substrates have long been thought to play a role in PD psychosis, they form the basis for several current therapeutic strategies of PD psychosis and merit continued attention. This section will review the importance of the cholinergic and serotonergic systems and other neuropsychiatric features commonly associated with PD psychosis.

2.1. Cholinergic System. The role of the central cholinergic system in PD psychosis is underscored by its involvement in cognition and sleep, two neuropsychiatric areas that are intricately linked to hallucinations and delusions in PD. PD dementia and dementia with Lewy bodies exhibit pronounced frontal cortical denervation due to disruption of the ascending cholinergic transmitter system and degeneration of central cholinergic structures involved in attention, cognition, and REM sleep such as the nucleus basalis of Meynert and pedunculopontine nucleus [41, 42]. In addition, pharmacological interventions with anticholinergic medications such as scopolamine or trihexyphenidyl are recognized to cause confusion in $\mathrm{PD}[16,43]$.

Cognitive impairment and dementia have been associated with PD psychosis in many studies [2, 23, 44]. Presence of hallucinations is a significant predictor of dementia in $\mathrm{PD}$, and cognitive decline is faster in those PD patients with hallucinations $[45,46]$. More recently, clinical trials in PD dementia, Alzheimer's disease, and dementia with Lewy bodies have demonstrated a positive effect of cholinesterase inhibitors on hallucinations and psychosis [47]. A subanalysis of $188 \mathrm{PD}$ hallucinators from a large, multicenter, double-blind, placebo-controlled trial of rivastigmine in mild to moderate PD dementia was conducted [47], and rivastigmine-placebo differences for several measures (i.e., ADAS-cog, ADCS-CGIC, and NPI-10) were found to be significantly larger in the hallucinators than in the nonhallucinators. Greater therapeutic benefit could be potentially derived from the use of cholinesterase inhibitors in select PD patients with dementia and hallucinations [48].

Sleep disturbances are frequent in PD patients, including those with hallucinations. Several studies suggest that PD hallucinations are related to sleep fragmentation and altered dream phenomena, but whether this represents a stepwise pattern or "continuum" [27], distinct but related factors [49], or predictors of future hallucinations $[49,50]$ is uncertain. Compared to PD patients without hallucinations, the hallucinators have decreased sleep efficiency, total REM sleep time, and REM sleep percentage on polysomnography [51-53] and altered circadian rest-activity rhythms on actigraphy [33]. These disturbances in sleep architecture, REM sleep, and circadian patterns suggest involvement of brainstem and hypothalamic sleep centers with complex interactions among neurotransmitters such as acetylcholine, serotonin, noradrenaline, histamine, GABA, hypocretin as well as dopamine [54]. Few PD studies, to date, have assessed the effect of antipsychotics on sleep and hallucinations. In a small, pilot study, Fernandez et al. assessed quetiapine's efficacy in improving visual hallucinations and its effect on sleep using polysomnography [55]. No significant differences in REM duration were found in either the quetiapine or placebo groups, although the sample size was small and study completion rate was low. The control of sleep-wake patterns integrates many neurotransmitters involved in $\mathrm{PD}$, and sleep and circadian disturbances may represent important targets not only for PD sleep but also for hallucinations.

2.2. Serotonergic System. The serotonin (5HT) system has been implicated in PD psychosis as well as in mood disturbances such as depression and anxiety. While an imbalance in serotonin and dopamine contributing to PD psychosis was suggested as early as 1975 by Birkmayer and Riederer [56], the development of antipsychotics with greater serotonergic affinity including 5HT-2a receptors, such as second-generation antipsychotics, clozapine, or quetiapine, has brought renewed attention to the serotonin system. In $\mathrm{PD}$, there is an extensive loss of serotonergic raphe neurons and a reduction of serotonergic projections to the frontal cortex, temporal cortex, and putamen. Complex interactions among dopamine, serotonin, acetylcholine, and norepinephrine, among others occur. Dopamine administration may lead to hyperstimulation of 5HT-2a receptors that affects glutamatergic-modulated activity of dopamine neurons in the ventral tegmental area; this may lead to excitation of the limbic system and inhibition of the prefrontal cortex [9], areas important to cognitive and behavioral processes. Additional evidence for serotonin's contribution to PD psychosis stems from a recent positron emission tomography study using a selective 5HT-2a receptor ligand, $\left[{ }^{18} \mathrm{~F}\right]$-setoperone, in 7 nondemented PD patients with visual hallucinations compared to 7 age-matched PD without visual hallucinations [57]. In this pilot study, the PD hallucinators had increased 5HT-2a receptor binding in multiple brain regions including the ventral visual pathway, bilateral dorsolateral prefrontal cortex, medial orbitofrontal cortex, and insula, suggesting alterations in pathways mediating visual and cognitive processing and a role for $5 \mathrm{HT}-2 \mathrm{a}$ in PD hallucinations. Furthermore, abnormalities in brain 5HT2a receptors were found in a recent postmortem tissue study [58]; increased $\left[{ }^{3} \mathrm{H}\right]$-ketanserin binding in the inferolateral temporal cortex was found in PD patients who had visual 
hallucinations, compared to those who did not, though potential confounders such as dementia, mood disorders, medications, and disease duration await further study.

PD psychosis and mood disorders both involve the serotonin system, and this relationship has been explored in some studies. Several reports demonstrate positive effects of antidepressants on PD psychosis [59-61]. However, the use of antidepressants in PD psychosis has been historically controversial as there also have been a few case reports of antidepressants (e.g., bupropion, fluoxetine, and mirtazapine) exacerbating PD psychosis [62-64]. The worsening of psychosis has been attributed to enhanced dopamine release by stimulation of serotonin receptors by the antidepressants. Despite this, antidepressants, particularly serotonin reuptake inhibitors (SSRIs) or serotonin-norepinephrine reuptake inhibitors (SNRIs), have been widely used in mood disturbances in PD without clear evidence of triggering or aggravating psychosis. In a review of depressed PD patients from our movement disorders clinic, the addition of SSRIs did not exacerbate hallucination risk, as measured by changes in thought disorder scores from the Unified Parkinson's disease Rating Scale, in depressed PD patients on stable dopaminergic medication regimens [65].

Several case reports and a small case series have suggested a beneficial effect of antidepressants in PD psychosis, particularly those patients with coexisting mood disorders [59-61]. Voon et al. reported a series of 10 PD patients with psychotic symptoms and comorbid depression or anxiety who were treated with antidepressants (citalopram or venlafaxine) and had pre- and posttreatment evaluations of their psychosis and mood disorders, in a prospective but unblinded fashion. Psychotic symptoms improved in $8 / 10$ patients when antidepressants were used either as monotherapy or as adjuncts to baseline antipsychotics (in 2/10). Proposed mechanisms for the positive effects of antidepressants on PD psychosis included the following: (1) improvement in underlying depression which could have exacerbated psychotic symptoms; (2) increased serotonergic tone since low serotonin levels can be associated with depression, aggression, and impulsivity; (3) restoration of serotonin-dopamine imbalance; (4) decrease in the upregulation of postsynaptic serotonin receptors due to raphe nuclei degeneration; or (5) REM suppression by the antidepressants $[60,61,66]$. Limitations, however, included a small, openlabel design, disease-related, and treatment-related differences among patients and heterogeneity of hallucinations, psychosis, and psychiatric diagnoses.

Despite the limitations and lack of controlled studies of antidepressants and PD psychosis, these reports raise questions regarding interactions between serotonindopamine systems, the relationship between hallucinations, psychosis and mood disorders in $\mathrm{PD}$, and the occurrence of psychotic depression in PD and how this differs from psychotic depression in other populations. Well-designed, controlled studies of select SSRIs or other antidepressants will be needed to determine if they may be an appropriate pharmacological strategy for treating psychosis in PD with or without comorbid mood disorders. Treatment rationales that incorporate comorbid neuropsychiatric phenomena such as cognition, sleep, and mood may provide alternative treatment strategies for PD psychosis.

\section{Neuroimaging: A Window into PD Psychosis}

Neuroimaging studies permit in vivo investigations of brain alterations in PD patients with hallucinations. Techniques utilized include structural magnetic resonance imaging (MRI) to assess gray matter atrophy, functional MRI to examine activation patterns, and perfusion scans to investigate changes in regional cerebral blood flow or glucose metabolism. The neuroimaging studies of PD and visual hallucinations provide clues as to underlying neuroanatomical substrates and pathophysiology of PD hallucinations.

3.1. Structural MRI. Structural MRI studies of visual hallucinations in PD have examined regional and global brain atrophy patterns. Using whole-brain voxel-based mophometry (VBM) to study cerebral atrophy, Ramírez-Ruiz et al. found significantly reduced gray matter volume in the lingual gyrus and superior parietal lobe, regions involved in higher-order visual processing, in the PD hallucinators, compared to nonhallucinating PD and healthy controls [40]. In another study, hippocampal atrophy was detected in 3 groups of PD patients (i.e., PD with dementia, nondemented PD with hallucinations, and nondemented PD without hallucinations), compared to healthy controls, at rates of $78 \%, 31 \%$, and $26 \%$, respectively, [35]. The nondemented, hallucinating PD group exhibited greater gray matter reduction in the hippocampal head, an area associated with specific memory functions. Of note, although the hallucinating PD patients were not demented, they performed worse on neuropsychological tests. The same authors studied 12 nondemented, hallucinating PD patients, compared to $14 \mathrm{PD}$ patients without hallucinations, and 12 healthy controls for a mean 29.91 (5.74) months with MRI (VBM analyses) and neuropsychological tests [67]. At follow-up, $75 \%$ of the hallucinating PD had developed dementia. The PD hallucinators demonstrated widespread limbic, paralimbic and neocortical gray matter loss, regions also abnormal in PD dementia. These studies support regional neuroanatomical changes and clinical links between hallucinations and cognitive impairment in PD.

3.2. Functional MRI. Functional MRI (fMRI) studies in PD patients with hallucinations demonstrate altered cortical activation patterns compared to PD nonhallucinators. Stebbins et al. studied $12 \mathrm{PD}$ patients with chronic visual hallucinations, matched for age, disease duration, and dopaminergic drug exposure to PD patients who had never hallucinated using two visual stimulation fMRI paradigms (i.e., stroboscopic and kinematic). The PD hallucinators had significantly greater frontal and subcortical activation to both visual stimulation paradigms and decreased cerebral activation in occipital, parietal, and temporal-parietal regions, compared to the nonhallucinators [22], thereby suggesting a disruption in normal visual processing mechanisms in the hallucinators. Another study using complex visual stimuli 
(e.g., face recognition task) revealed significant reductions in right prefrontal areas including the inferior, superior, and middle frontal gyrus and anterior cingulate gyrus in PD hallucinators to the face stimulus, compared to nonhallucinating PD and healthy controls [68]. These findings suggest disruptions between anterior (e.g., frontal) and posterior (e.g., parietal, temporal, occipital) regions that are important in processing visual stimuli and impaired abilities to distinguish relevant from irrelevant visual information in PD hallucinators. Further evidence for impaired "bottomup" visual processing in hallucinating PD comes from a fMRI study by Meppelink et al. in which several seconds prior to image recognition task, the nondemented, hallucinating PD patients showed reduced activation of the lateral occipital cortex and extrastriate temporal visual cortices, compared to nonhallucinating PD and healthy controls [69]. In contrast to other studies, however, the authors did not find increased "top-down" frontal activation in their sample.

3.3. Metabolic Studies. Decreased perfusion or glucose metabolism in predominantly posterior brain regions has been reported in PD hallucinators using single photon emission computed tomography (SPECT) or positron emission tomography (PET) modalities. Using 99mTc-HMPAO SPECT, Okada et al. found decreased cerebral blood flow to the left temporal lobe and temporal-occipital lobe regions in hallucinating PD patients [70]. In one $\left.{ }^{123} \mathrm{I}\right]$ IMP SPECT study, hypoperfusion in the right fusiform gyrus but hyperperfusion in the right superior and middle temporal gyri was found in PD hallucinators, when covarying for MMSE score and PD duration [71], suggesting importance of the visual ventral stream. Also using [ $\left.{ }^{123} \mathrm{I}\right]$ IMP SPECT scans, Matsui et al. found reduced perfusion in bilateral inferior parietal lobules, inferior temporal gyrus, precuneus gyrus, and occipital cortex in $31 \mathrm{PD}$ patients with visual hallucinations, compared to 39 without hallucinations [38]. Decreased metabolism in temporal-occipital-parietal regions was found in $8 \mathrm{PD}$ hallucinators compared to 11 nonhallucinators with $\left[{ }^{18} \mathrm{~F}\right]$ FDG-PET [34]. Similar to some fMRI results which indicate disruptions in frontal and posterior activation patterns, Nagano-Saito et al. found greater regional cerebral glucose metabolic rates in frontal regions, especially the left superior frontal gyrus, in 8 nondemented, PD patients with visual hallucinations, compared to nonhallucinating PD and healthy controls, using $\left[{ }^{18} \mathrm{~F}\right]$ FDG-PET [72]. Overall, neuroimaging studies using different modalities emphasize the relationship between frontal and posterior brain regions, visual and cognitive processing, and pathogenesis of $\mathrm{PD}$ hallucinations.

3.4. Challenges. While the neuroimaging studies allow for an in vivo assessment of the brain in PD hallucinators, there are several limitations, confounders, and challenges. Acquiring neuroimaging studies in PD patients can be difficult due to issues of motor fluctuations, tremor or dyskinesias, impaired attention, dementia, and active psychosis. Moreover, fMRI studies in particular can be affected by factors such as impaired attention, motivation, and cognition; medication effects including antipsychotics; and visual acuity deficits. Although interesting, imaging PD patients while they are actively hallucinating or delusional could pose certain challenges. The majority of the neuroimaging studies, to date, have focused on chronic visual hallucinations, and thus, whether the findings reflect the pathogenesis of delusions or hallucinations in other sensory modalities is not known. Additionally, comorbid neuropsychiatric issues such as cognitive impairment, even mild, and depression may share neuroanatomical substrates (e.g., temporal lobe) and could influence interpretations of studies. Despite these challenges, studies using different neuroimaging modalities, applied individually or in combination, have the potential to enhance our understanding of the pathogenesis of $\mathrm{PD}$ psychosis.

\section{Genetic Risk Factors for PD Psychosis}

Besides clinical risk factors such as age, PD duration and severity, depression, cognitive impairment and dementia, and sleep disturbances $[2,3]$, genetic factors may be involved in the development of PD psychosis. Genetic polymorphisms that affect levodopa or dopamine-agonist metabolism have been shown to play a role in other medication-related complications such as dyskinesias or motor fluctuations $[73,74]$. With the growing field of pharmacogenetics, interindividual differences in medication responses due to underlying genetic polymorphisms may become increasingly important in drug development, evaluation of drug efficacy and toxicity, and ultimately, individualized patient care.

Various genetic polymorphisms have been considered regarding increased risk of psychosis in PD. This section will discuss several studies of genetic polymorphisms in the dopamine, serotonin, and cholecystokinin systems as well as apolipoprotein E (APOE) 4 allele status in PD hallucinators. In general, many of the studies have yielded conflicting results, and thus, the relationship between genetic interindividual variability and disease-related complications of PD awaits further exploration. Differences in methodologies and ethnic backgrounds of subjects may be critical contributions to the negative or conflicting study results.

4.1. Dopaminergic System. Investigations of the dopaminergic system have included D1 class receptors (DRD1 and DRD5), D2 class receptors (DRD2, DRD3, DRD4), as well as the dopamine transporter gene (DAT). Makoff et al. studied polymorphisms of DRD2 $(-141 \mathrm{C} / \mathrm{del}$ in the promoter region and TaqIA restriction fragment length polymorphism $\mathrm{C}>\mathrm{T}$ ) and DRD3 (Ser9Gly) in hallucinating and nonhallucinating white PD patients [75]. No association of these DRD2 or DRD3 polymorphisms was found when comparing the 84 hallucinators to the 71 nonhallucinators as a group. However, an association was found with the $\mathrm{C}$ allele of the TaqIA polymorphism to DRD2 and late-onset hallucinators (i.e., those PD patients who developed hallucinations after 5 years of disease). The nonhallucinating controls for the whole group were matched for disease duration, age at disease onset, duration of dopaminergic therapy, and gender, but not medication doses or classes of dopaminergic agents. 
In a case-control study of 44 matched pairs of white PD patients with and without chronic hallucinations, Goetz et al. assayed dopamine receptor genes, including DRD1, DRD2, and DRD3 [76]. They found no significant difference in allele frequencies or distribution of genotypes between hallucinating and nonhallucinating PD patients for DRD1 and DRD3, although the DRD3 2 allele had a borderline increased frequency in hallucinators $(P=.047)$. Similarly, a case-control study of Chinese PD patients with and without hallucinations did not find an association at DRD2 (TaqIA, $32806 \mathrm{C}>\mathrm{T}$ ), DRD3 (Ser9Gly and Msp1), or DRD5 (978 $\mathrm{T}>\mathrm{C}$ ) [77]. Of note, the DRD2 polymorphism has been associated with dyskinesias and wearing off in $\operatorname{PD}[78,79]$. The positive association of the DRD2 polymorphism with dyskinesias and wearing off but not with hallucinations suggests that the underlying pathogenesis of neuropsychiatric and motor complications in PD differs. Alternatively, polymorphisms in the dopamine receptor genes may be minimally involved in PD psychosis, given the negative results from the three studies described.

Other studies have focused on the dopamine transporter gene (DAT) since the dopamine transporter controls the presynaptic reuptake of dopamine. In some reports, a $40 \mathrm{bp}$ variable number of tandem repeat (VNTR) polymorphism in the $3^{\prime}$-untranslated region of the DAT gene has been associated with psychiatric conditions such as attentiondeficit hyperactivity disorder, alcoholism, and schizophrenia. In a study of white PD patients, Kaiser et al. examined the VNTR polymorphism of the DAT gene, along with DRD2, DRD3, and DRD4 polymorphisms, and found that the $9 \times$ 40 bp VNTR allele of the DAT gene was more frequently present in those levodopa-treated PD patients with psychosis or dyskinesias, compared to nonaffected patients (odds ratio 2.6; 95\% CI: $1.3-5.3 ; P=.008$ and odds ratio 2.5 ; $95 \% \mathrm{CI}$ : $1.3-4.7 ; P=.007$, respectively, for psychosis and dyskinesia) [80]. The finding of differences in the 9-copy allele of the DAT VNTR, however, was not confirmed in either a casecontrol study of Chinese PD patients with and without hallucinations [77] or in our case-control study of white PD patients with and without hallucinations [81]. Larger studies of different ethnic populations are needed to further study the role of the DAT gene and dopaminergic system in PD psychosis.

4.2. Serotoninergic System. Polymorphisms in the serotonin (5HT) transporter gene have been thought to influence anxiety and depression by altering serotonergic tone $[82,83]$. The serotonin transporter gene, similar to the dopamine transporter gene, plays a critical role in the termination of 5HT transmission by controlling presynaptic reuptake [84]. Two functional polymorphisms in the serotonin transporter gene have been identified and contribute to protein expression. These include a promoter VNTR (short, long alleles) and intron $2 \operatorname{VNTR}(9,10,11,12$ alleles). Less efficient transcription and decreased reuptake results from a deletion (short [s] allele compared to long [1] allele) or presence of 10-copy allele in intron 2 VNTR in the transporter gene. Some studies have reported a significant association of the s/s and $1 / \mathrm{s}$ genotypes and anxiety-related traits in the general population as well as in depressed or anxious PD patients [82]. In a study of 32 genotyped PD patients who were administered the Hamilton Depression and Anxiety scales, those PD patients who had the short allele had significantly higher scores on the depression and anxiety measures [85]. For the VNTR element in intron 2 of the serotonin transporter gene in which four variants may occur, increased frequency of the 9-copy allele has been reported in affective disorders [83]. However, this finding was not confirmed in PD patients with depression [86]. In our case-control study, we examined these two serotonin transporter gene polymorphisms in white PD patients with and without hallucinations but did not find any differences in allelic or genotypic frequencies of short or long alleles or intron sequences of the VNTR element [87]. Thus, the serotonin transporter gene polymorphisms may have greater association with anxiety and other affective disorders rather than psychosis, although further study is needed. Alternatively, since the serotonin system encompasses many receptor subtypes, individual receptors rather than the transporter itself may be associated with psychosis, as suggested by atypical antipsychotic pharmacology. Kiferle et al. examined polymorphisms in the 5HT-2a gene (T102C) as well as the serotonin transporter gene (short, long alleles) [88]. However, in their nondemented, white PD group, no significant differences in either polymorphism between PD patients with and without hallucinations were found.

4.3. Cholecystokinin. Cholecystokinin (CCK), a neuropeptide found in both the gut and central nervous system, is involved in dopaminergic regulation and colocalizes with dopaminergic neurons. The CCK receptor genes, CCKAR and CCKBR, have been cloned and sequenced in humans [89]. Centrally, CCKAR mediates the behavioral actions of CCK and CCK-stimulated dopamine release in the posterior nucleus accumbens. In contrast, CCKBR mediates CCK inhibition of dopamine release in the anterior nucleus accumbens [90]. The nucleus accumbens, part of the cortico-striato-thalamo-cortical loop, is associated with the mesolimbic pathway and behaviors such as reward, pleasure, and addiction. Due to their role in mesocorticolimbic circuitry, CCK and its receptors were initially studied in alcoholism and schizophrenia with auditory hallucinations and later in PD psychosis.

Studies in Asian PD patients suggest that CCK polymorphisms may differ in hallucinating PD patients. Fujii et al. reported that Japanese PD patients with hallucinations differed at the CCK $-45 \mathrm{C} / \mathrm{T}$ locus, with overrepresentation of CT and TT genotypes in the hallucinators, though this did not remain significant when correcting for multiple comparisons [91]. Since the $\mathrm{C}$ to $\mathrm{T}$ transition occurs in the Sp1-binding cis element of the CCK gene promoter, mutations at this site might affect promoter activity and gene transcription [92]. In a study of Chinese hallucinating and nonhallucinating PD patients treated with levodopa, the hallucinators significantly differed at the CCK $-45 \mathrm{C} / \mathrm{T}$ locus, with overrepresentation of the $\mathrm{T}$ allele in the hallucinators [93]. Moreover, there was an almost 6-fold increased risk for 
developing hallucinations if the CCK T allele and CCKAR C allele were present in combination. In our investigation of the CCK system in a matched sample of white PD hallucinating cases and nonhallucinating controls, we did not find any difference in allele frequencies or genotype distribution for CCK but detected a trend for an over-representation of the CCK T allele in hallucinating PD patients $(P=.06)$ [94].

4.4. APOE4. The APOE4 allele is recognized as an important genetic risk factor in Alzheimer's disease but has been variably reported in PD dementia. Because dementia and psychosis may coexist, polymorphisms in APOE4 have been investigated as genetic risk factors for PD hallucinations. In a study of nondemented PD patients, the APOE4 allele was present in $13 / 17(76 \%)$ hallucinators compared to 20/88 (23\%) nonhallucinators, remaining significant after adjustment for age, PD severity, treatment duration, levodopa dose, and agonist treatment [95]. Other studies, however, have failed to replicate these findings. Whether or not the APOE4-positive, hallucinating patients subsequently developed dementia or had coexisting Alzheimer's disease pathology is not known. Goetz et al. did not find any difference in APOE4 alleles in their case-control study, despite the PD hallucinators having lower Mini-mental State examination scores (mean [SD] for hallucinators 23.7 [5.3] and nonhallucinators 28.8 [2.4]) [76]. No association between APOE4 and PD hallucinations was found in a study of 47 autopsy-proven PD patients [96]; in this study, Camicioli et al. included patients as "hallucinators" if hallucinations were ever recorded in the charts regardless of persistence or etiology. While even transient hallucinations may not be "benign" in their course, it remains to be seen whether genetic susceptibilities differ in PD patients with illusions, intermittent hallucinations, chronic hallucinations, or delusions.

4.5. Challenges. Conflicting results and methodological issues have complicated the studies of genetic polymorphisms and PD hallucinations. Confounding variables that may influence these genetic association studies include but are not limited to small sample sizes, methodological differences (case-control studies versus larger population-wide studies), and differences in demographic or PD-related issues such as age, gender, disease duration, medications, cognitive, and mood disorders. Moreover, an important consideration is that genetic polymorphisms vary greatly among different ethnic groups. In addition, it remains possible that some nonhallucinating patients examined in cross-sectional studies might convert to hallucinators over time. Longitudinal follow-up would be necessary to assess conversion risk and genetic status as a predictor of hallucinations. Nevertheless, the genetic association studies may prove to be informative and useful regarding genetic susceptibility, drug development, and potentially individualized treatment regimens.

\section{Animal Models of Psychosis}

Animal models provide important translational tools by which to study the neurobiological substrates of disease and to investigate treatments for specific disease-related symptoms. Animal models such as the 1-methyl-4-phenyl1,2,3,6-tetrahydropyridine- (MPTP-) lesioned primates and 6-hydroxydopamine- (6-OHDA-) lesioned rodents have been influential in studying parkinsonism and levodopainduced dyskinesias. However, hallucinations and other psychotic behavior have been more difficult to study in animals given the subjective nature of the hallucinatory experience and the challenge of inferring meaning from an animal's behavior. In rodents and primates, amphetamine and other stimulants (e.g., the glutamate NMDA receptor antagonists ketamine and phencyclidine) have been long used as potential models of psychosis or positive symptoms of schizophrenia due to their ability to stimulate locomotor activity, produce stereotypies, and enhance striatal dopamine release [97]. In primates treated with chronic amphetamine, induced movements such as stereotypies and hyperactivity and behaviors such as hypervigilance, checking, grasping, and staring may occur $[98,99]$.

\subsection{Studies of Psychosis-Like Behaviors in MPTP-Lesioned} Primates. MPTP-lesioned primates have been noted to experience abnormal psychomimetic behaviors that are reminiscent of the "psychosis-like" behaviors in the amphetaminesensitized animals, and these behaviors may have utility in studying PD psychosis [100, 101]. Fox et al. examined these phenomena in primates; marmosets treated with MPTP and housed under controlled conditions were subsequently exposed, after a stable parkinsonian period, to several different dopaminergic and antipsychotic medications. In order to quantify the psychosis-like behaviors, the authors proposed a rating scale with behavioral categories including: (1) agitation, hyperkinesias; vocalizations; (2) hallucinatorylike response to apparent nonstimuli, tracking, staring; (3) obsessive grooming, scratching or grooming repetitively; and (4) stereotypes, repetitive side-to-side jumping, head checking movements, purposeless running, and grasping at the bars [101]. These behaviors, deemed distinct from dyskinesias, were rated by a blinded observer, in addition to parkinsonism and dyskinesias, and summed for a total psychosis-like behavior score.

In a series of experiments, the authors examined responses to various dopaminergic medications and antipsychotics. Both levodopa and dopaminergic receptor agonists (pergolide, pramipexole, and ropinirole) induced peak-dose psychosis-like behavior in the MPTP-lesioned primates as well as reversed parkinsonian disability, compared to the vehicle [100]. No difference in psychosis-like behavior was observed among the dopaminergic agents. In a companion study using a similar protocol [101], the authors found that levodopa produced psychosis-like behavior in the 7 marmosets studied. Of the psychosis-like behaviors exhibited, stereotypies were the most common, and all animals exhibited staring and tracking behavior (hallucinatorylike). There was no correlation between severity of dyskinesias and psychosis-like behavior in individual animals with levodopa. Amantadine coadministered with levodopa significantly increased total peak-dose psychosis-like behavior but reduced peak-dose dyskinesias. Psychosis-like 
behaviors, particularly stereotypies, were decreased when haloperidol was coadminstered with levodopa. Not surprisingly, haloperidol, compared to vehicle, worsened parkinsonism and reduced dyskinesias. Although quetiapine was coadministered with levodopa in only 6 animals, results differed based on dose; significant reduction in the total psychosis-like score was seen in the $1.5 \mathrm{mg} / \mathrm{kg}$ group but not the $0.5 \mathrm{mg} / \mathrm{kg}$ or $4.5 \mathrm{mg} / \mathrm{kg}$ doses, compared to vehicle. Clozapine reduced psychosis-like behaviors, compared to vehicle, with a decrease in hallucinatory-like behavior. Neither quetiapine nor clozapine worsened parkinsonism or produced somnolence.

5.2. Challenges. One of the greatest challenges of the animal models is whether one can infer that the primates' behaviors recapitulate the human experiences of hallucinations and psychosis. However, these dopaminergic-stimulated abnormal behaviors, as distinguished from dyskinesias, may represent a close approximation of PD neuropsychiatric disturbances and an opportunity to evaluate pathophysiology and therapeutics for psychosis and heightened dopaminergic states.

\section{Recent Pharmacological Trials and Challenges of PD Psychosis Trials}

Atypical antipsychotics, such as clozapine and quetiapine, which have greater serotonergic antagonism than dopamine receptor blockade have been generally favored in the management of PD psychosis due to the decreased risk of worsened parkinsonism and other extrapyramidal syndromes. Much of the serotonin effect of these atypical antipsychotics relates to drug interactions with the 5HT-2a receptors, which in the central nervous system can be found in the cortex, basal ganglia (caudate and putamen), and hippocampus [102]. Interestingly, 5HT-2a antagonists or inverse agonists including clozapine also have demonstrated improvement in parkinsonian motor function in primates and humans $[103,104]$. Based on these pharmacological profiles, several atypical antipsychotics acting on the 5HT-2a system, such as melperone and pimavanserin (ACP-103), have been recently studied in PD psychosis. The following section will briefly discuss findings related to these newer agents and challenges of PD psychosis trials. Other medications studied in PD psychosis, including quetiapine, clozapine, among other atypical antipsychotics, have been the subject of other recent reviews and are not discussed here [105].

6.1. Melperone. Melperone has been available in some European countries and in use as an antipsychotic for schizophrenia for over 10 years. Although a butyrophenone, melperone has been considered an atypical antipsychotic due to its low extrapyramidal symptoms and lack of increase in plasma prolactin $[106,107]$. It is similar to clozapine in that it also has high 5HT-2a affinity relative to dopamine (D2) receptor binding affinities. In an open-label trial for PD psychosis, Barbato et al. assessed the clinical efficacy and safety of melperone in $30 \mathrm{PD}$ patients with psychosis over a 24-month period [108]. In 28/30 PD patients, psychotic symptoms, as measured by the Brief Psychiatric Rating Scale (BPRS), improved with a mean daily dose of $37.5 \mathrm{mg}$ (range $12.5-75 \mathrm{mg}$ daily) without worsening motor scores. Doses were much smaller than those utilized in schizophrenia, similar to the low clozapine and quetiapine doses used in PD psychosis. Side effects included hypotension, dizziness, and sedation (leading to discontinuation by 2 patients). Based on this study, a multicenter, randomized, doubleblind, placebo-controlled, 10-week, Phase II trial evaluating the safety and efficacy of melperone in PD psychosis was conducted in the US and recently finished. However, to date, no results have been published in scientific journals.

6.2. Pimavanserin (ACP-103). Pimavanserin (ACP-103) is a 5HT-2a receptor inverse agonist, which has been recently investigated in PD psychosis $[109,110]$. Although pimavanserin (ACP-103) also has some 5HT-2c receptor affinity, it exhibits about 30 -fold greater selectivity for the 5HT-2a receptor compared to the 5HT-2c receptor [103]. Results from a multicenter, randomized, double-blind, placebocontrolled, 28-day, Phase II study to evaluate the tolerability, safety, and efficacy of pimavanserin in $60 \mathrm{PD}$ patients were recently published [111]. The study, powered for differences in the UPDRS Parts II and III scores, had favorable results regarding motor function, with no significant differences observed between drug and placebo groups. In addition, there were no significant differences in adverse events between treatment groups. Regarding psychosis outcomes, the Scale for the Assessment of Positive Symptoms (SAPS) total domain score was chosen as the primary outcome measure for efficacy based on its use in the Parkinson's Study Group clozapine trial, and a trend for greater improvement in pimavanserin-treated patients was detected $(P=.09$, effect size $=0.52$ ). Analyses of many individual items from the SAPS and UPDRS psychosis question failed to reveal significant differences between treatment groups; however, the global ratings for hallucinations and delusions significantly favored the pimavanserin arm. Subsequently, a multicenter, randomized, double-blind, placebo-controlled, Phase III trial with a larger sample of PD patients was completed; reports reveal that although the drug was tolerated in terms of motor function and side effects, a statistically significant benefit for psychosis measures was not achieved [112]. Of note, this same compound has been under evaluation in primate models of levodopa-induced dyskinesias [103] and schizophrenia-related psychosis and insomnia. Since serotonin may influence sleep architecture, pimavanserin and similar compounds could also improve sleep maintenance and quality in those with insomnia $[109,113]$.

6.3. Challenges. In addition to the pharmacological challenges of finding antipsychotics that safely treat PD psychosis without worsening parkinsonism or causing other adverse effects, there are challenges related conducting the trials and many issues pertaining to optimal trial design, inclusion/exclusion criteria, subject recruitment, and study completion. In 2006, the Quality Standards Subcommittee 
of the American Academy of Neurology published a Practice Parameter report on the evaluation and treatment of depression, psychosis, and dementia in PD. Of 23 articles on psychosis treatment in PD patients identified by the authors, only four Class I and II articles were accepted for review. Based on a review of these PD psychosis studies on clozapine, quetiapine, and olanzapine, the authors concluded that, as demonstrated by one Class I study [114] (PSG) and one Class II study [115], clozapine was probably an effective treatment for PD psychosis without motor worsening. Clozapine use, despite its efficacy, is often limited in clinical practice due to the risk of fatal agranulocytosis and requirement for neutrophil count monitoring. Only one Class II study [115] demonstrated that quetiapine possibly improved PD psychosis. Olanzapine failed to improve psychosis and worsened parkinsonism [116, 117]. As such, the Practice Parameter authors recommended that clozapine could be considered as Level B and quetiapine, as Level C treatments, for PD psychosis [118]. The conclusions from the AAN Practice Parameter underscore the need to identify novel compounds that are safe and effective in reducing psychosis without compromising motor function. Furthermore, these agents will require evaluation in well-designed, randomized, controlled trials in order to withstand rigorous methodological review and meet criteria for evidence-based medicine. Two doubleblind, placebo-controlled studies, subsequently published and thus not included in the AAN review, have failed to demonstrate significant improvement with quetiapine in ratings of psychosis although parkinsonism did not worsen $[119,120]$. The quetiapine studies, however, have been criticized for methodological issues, such as poor recruitment, patient populations with mixed diagnoses (some with $\mathrm{PD}$, PD and dementia, dementia with Lewy bodies, or Alzheimer disease with parkinsonism), low doses of medications, selection of psychosis rating scales or primary outcomes, and small sample sizes. Recruitment for PD psychosis clinical trials remains a challenging issue; influential factors include the widespread clinical use of quetiapine despite negative double-blind, placebo-controlled trial results; patient and caregiver concerns about placebo assignment especially when hallucinations are frightening and delusions are present; risk of worsened motor function; and recent "black box" warnings by the FDA regarding increased risk of death in elderly patients with dementia who received treatment with antipsychotics. These important issues also must be addressed in trials for PD psychosis.

\section{Conclusions and Future Directions}

Although the dopaminergic system remains important in the pathogenesis of psychosis in PD, new research strategies should consider the complex relationships among other neurochemical and neuroanatomical substrates and potentially integrate these elements into targets for drug development. Similarly, investigations of PD psychosis should examine the influences of other neuropsychiatric features of PD such as attention, cognition, sleep, and mood. Alterations in visual and cognitive processing may provide useful targets, as deficits span from the retina to brainstem to cortical regions.
While the optimal treatment of PD psychosis remains a challenge, integrating studies of neuroimaging and genetic susceptibility, and perhaps, animal models may permit advances in drug development. Examination of clinical and genetic factors of those PD patients who never hallucinate despite high dopaminergic medication doses may also provide useful information on protective mechanisms and strategies.

\section{Acknowledgments}

Dr. Goldman has received research support from NIH K23060949 and the Parkinson's Disease Foundation.

\section{References}

[1] G. Fenelon and G. Alves, "Epidemiology of psychosis in Parkinson's disease," Journal of the Neurological Sciences, vol. 289, no. 1-2, pp. 12-17, 2010.

[2] G. Fenelon, F. Mahieux, R. Huon, and M. Ziégler, "Hallucinations in Parkinson's disease. Prevalence, phenomenology and risk factors," Brain, vol. 123, no. 4, pp. 733-745, 2000.

[3] J. R. Sanchez-Ramos, R. Ortoll, and G. W. Paulson, "Visual hallucinations associated with Parkinson disease," Archives of Neurology, vol. 53, no. 12, pp. 1265-1268, 1996.

[4] B. Ravina, K. Marder, H. H. Fernandez et al., "Diagnostic criteria for psychosis in Parkinson's disease: report of an NINDS, NIMH Work Group," Movement Disorders, vol. 22, no. 8, pp. 1061-1068, 2007.

[5] C. G. Goetz and G. T. Stebbins, "Risk factors for nursing home placement in advanced Parkinson's disease," Neurology, vol. 43, no. 11, pp. 2227-2229, 1993.

[6] C. G. Goetz and G. T. Stebbins, "Mortality and hallucinations in nursing home patients with advanced Parkinson's disease," Neurology, vol. 45, no. 4, pp. 669-671, 1995.

[7] D. Aarsland, J. P. Larsen, E. Tandberg, and K. Laake, "Predictors of nursing home placement in Parkinson's disease: a population-based, prospective study," Journal of the American Geriatrics Society, vol. 48, no. 8, pp. 938-942, 2000.

[8] S. Kuzuhara, "Drug-induced psychotic symptoms in Parkinson's disease. Problems, management and dilemma," Journal of Neurology, vol. 248, supplement 3, pp. III28-III31, 2001.

[9] E. C. H. Wolters, "Intrinsic and extrinsic psychosis in Parkinson's disease," Journal of Neurology, Supplement, vol. 248, supplement 3, pp. 22-27, 2001.

[10] E. C. H. Wolters, "PD-related psychosis: pathophysiology with therapeutical strategies," Journal of Neural Transmission, Supplement, no. 71, pp. 31-37, 2006.

[11] R. Holloway, I. Shoulson, K. Kieburtz et al., "Pramipexole vs Levodopa as initial treatment for Parkinson disease: a randomized controlled trial," Journal of the American Medical Association, vol. 284, no. 15, pp. 1931-1938, 2000.

[12] K. M. Biglan, R. G. Holloway, M. P. McDermott, and I. H. Richard, "Risk factors for somnolence, edema, and hallucinations in early Parkinson disease," Neurology, vol. 69, no. 2, pp. 187-195, 2007.

[13] P. A. LeWitt, K. E. Lyons, and R. Pahwa, "Advanced Parkinson disease treated with rotigotine transdermal system: PREFER Study," Neurology, vol. 68, no. 16, pp. 1262-1267, 2007. 
[14] O. Rascol, D. J. Brooks, A. D. Korczyn, P. P. De Deyn, C. E. Clarke, and A. E. Lang, "A five-year study of the incidence of dyskinesia in patients with early Parkinson's disease who were treated with ropinirole or levodopa," The New England Journal of Medicine, vol. 342, no. 20, pp. 1484-1491, 2000.

[15] U. K. Rinne, F. Bracco, C. Chouza et al., "Early treatment of Parkinson's disease with cabergoline delays the onset of motor complications. Results of a double-blind levodopa controlled trial," Drugs, vol. 55, supplement 1, pp. 23-30, 1998.

[16] Y. De Smet, M. Ruberg, and M. Serdaru, "Confusion, dementia and anticholinergics in Parkinson's disease," Journal of Neurology Neurosurgery and Psychiatry, vol. 45, no. 12, pp. 1161-1164, 1982.

[17] N. J. Diederich, C. G. Goetz, R. Raman, E. J. Pappert, S. Leurgans, and V. Piery, "Poor visual discrimination and visual hallucinations in Parkinson's disease," Clinical Neuropharmacology, vol. 21, no. 5, pp. 289-295, 1998.

[18] N. J. Diederich, C. G. Goetz, and G. T. Stebbins, "Repeated visual hallucinations in Parkinson's disease as disturbed external/internal perceptions: focused review and a new integrative model," Movement Disorders, vol. 20, no. 2, pp. 130-140, 2005.

[19] S. Holroyd, L. Currie, and G. F. Wooten, "Prospective study of hallucinations and delusions in Parkinson's disease," Journal of Neurology Neurosurgery and Psychiatry, vol. 70, no. 6, pp. 734-738, 2001.

[20] S. Holroyd and G. F. Wooten, "Preliminary fMRI evidence of visual system dysfunction in Parkinson's disease patients with visual hallucinations," Journal of Neuropsychiatry and Clinical Neurosciences, vol. 18, no. 3, pp. 402-404, 2006.

[21] V. Pieri, N. J. Diederich, R. Raman, and C. G. Goetz, "Decreased color discrimination and contrast sensitivity in Parkinson's disease," Journal of the Neurological Sciences, vol. 172, no. 1, pp. 7-11, 2000.

[22] G. T. Stebbins, G. G. Goetz, M. C. Carrillo et al., "Altered cortical visual processing in PD with hallucinations: an fMRI study," Neurology, vol. 63, no. 8, pp. 1409-1416, 2004.

[23] J. Barnes and L. Boubert, "Executive functions are impaired in patients with Parkinson's disease with visual hallucinations," Journal of Neurology, Neurosurgery and Psychiatry, vol. 79, no. 2, pp. 190-192, 2008.

[24] A. M. Meppelink, J. Koerts, M. Borg, K. L. Leenders, and T. van Laar, "Visual object recognition and attention in Parkinson's disease patients with visual hallucinations," Movement Disorders, vol. 23, no. 13, pp. 1906-1912, 2008.

[25] B. Ramírez-Ruiz, C. Junqué, M. J. Martí, F. Valldeoriola, and E. Toloso, "Neuropsychological deficits in Parkinson's disease patients with visual hallucinations," Movement Disorders, vol. 21, no. 9, pp. 1483-1487, 2006.

[26] B. Ramirez-Ruiz, C. Junque, M. J. Marti, F. Valldeoriola, and E. Tolosa, "Cognitive changes in Parkinson's disease patients with visual hallucinations," Dementia and Geriatric Cognitive Disorders, vol. 23, no. 5, pp. 281-288, 2007.

[27] I. Arnulf, A. M. Bonnet, P. Damier et al., "Hallucinations, REM sleep, and Parkinson's disease: a medical hypothesis," Neurology, vol. 55, no. 2, pp. 281-288, 2000.

[28] I. Arnulf, S. Leu, and D. Oudiette, "Abnormal sleep and sleepiness in Parkinson's disease," Current Opinion in Neurology, vol. 21, no. 4, pp. 472-477, 2008.

[29] C. L. Comella, “Sleep disorders in Parkinson's disease: an overview," Movement Disorders, vol. 22, no. 17, pp. S367S373, 2007.
[30] R. B. Postuma, J. F. Gagnon, M. Vendette, K. Charland, and J. Montplaisir, "Manifestations of Parkinson disease differ in association with REM sleep behavior disorder," Movement Disorders, vol. 23, no. 12, pp. 1665-1672, 2008.

[31] E. Sinforiani, C. Pacchetti, R. Zangaglia, C. Pasotti, R. Manni, and G. Nappi, "REM behavior disorder, hallucinations and cognitive impairment in Parkinson's disease: a two-year follow up," Movement Disorders, vol. 23, no. 10, pp. 14411445, 2008.

[32] E. Sinforiani, R. Zangaglia, R. Manni et al., "REM sleep behavior disorder, hallucinations, and cognitive impairment in Parkinson's disease," Movement Disorders, vol. 21, no. 4, pp. 462-466, 2006.

[33] D. L. Whitehead, A. D. M. Davies, J. R. Playfer, and C. J. Turnbull, "Circadian rest-activity rhythm is altered in Parkinson's disease patients with hallucinations," Movement Disorders, vol. 23, no. 8, pp. 1137-1145, 2008.

[34] H. Boecker, A. O. Ceballos-Baumann, D. Volk, B. Conrad, H. Forstl, and P. Haussermann, "Metabolic alterations in patients with Parkinson disease and visual hallucinations," Archives of Neurology, vol. 64, no. 7, pp. 984-988, 2007.

[35] N. Ibarretxe-Bilbao, B. Ramírez-Ruiz, E. Tolosa et al., "Hippocampal head atrophy predominance in Parkinson's disease with hallucinations and with dementia," Journal of Neurology, vol. 255, no. 9, pp. 1324-1331, 2008.

[36] H. Kataoka, Y. Furiya, M. Morikawa, S. Ueno, and M. Inoue, "Increased temporal blood flow associated with visual hallucinations in Parkinson's disease with dementia," Movement Disorders, vol. 23, no. 3, pp. 464-465, 2008.

[37] H. Matsui, K. Nishinaka, T. Miyoshi et al., "Thalamic hyperperfusion in verbal hallucination of Parkinsonian patients," Internal Medicine, vol. 46, no. 21, pp. 1765-1769, 2007.

[38] H. Matsui, K. Nishinaka, M. Oda et al., "Hypoperfusion of the visual pathway in parkinsonian patients with visual hallucinations," Movement Disorders, vol. 21, no. 12, pp. 2140-2144, 2006.

[39] B. Ramírez-Ruiz, M. J. Martí, E. Tolosa et al., "Brain response to complex visual stimuli in Parkinson's patients with hallucinations: a functional magnetic resonance imaging study," Movement Disorders, vol. 23, no. 16, pp. 2335-2343, 2008.

[40] B. Ramírez-Ruiz, M. J. Martí, E. Tolosa et al., "Cerebral atrophy in Parkinson's disease patients with visual hallucinations," European Journal of Neurology, vol. 14, no. 7, pp. 750-756, 2007.

[41] E. K. Perry, M. Curtis, and D. J. Dick, "Cholinergic correlates of cognitive impairment in Parkinson's disease: comparisons with Alzheimer's disease," Journal of Neurology Neurosurgery and Psychiatry, vol. 48, no. 5, pp. 413-421, 1985.

[42] R. M. Zweig, W. R. Jankel, J. C. Hedreen, R. Mayeux, and D. L. Price, "The pedunculopontine nucleus in Parkinson's disease," Annals of Neurology, vol. 26, no. 1, pp. 41-46, 1989.

[43] E. K. Perry and R. H. Perry, "Acetylcholine and hallucinations: disease-related compared to drug-induced alterations in human consciousness," Brain and Cognition, vol. 28, no. 3, pp. 240-258, 1995.

[44] D. Aarsland, K. Brønnick, U. Ehrt et al., "Neuropsychiatric symptoms in patients with Parkinson's disease and dementia: frequency, profile and associated care giver stress," Journal of Neurology, Neurosurgery and Psychiatry, vol. 78, no. 1, pp. 3642, 2007. 
[45] D. Aarsland, K. Andersen, J. P. Larsen, A. Lolk, and P. KraghSørensen, "Prevalence and characteristics of dementia in Parkinson disease: an 8-year prospective study," Archives of Neurology, vol. 60, no. 3, pp. 387-392, 2003.

[46] D. Aarsland, K. Andersen, J. P. Larsen et al., "The rate of cognitive decline in Parkinson disease," Archives of Neurology, vol. 61, no. 12, pp. 1906-1911, 2004.

[47] M. Emre, D. Aarsland, A. Albanese et al., "Rivastigmine for dementia associated with Parkinson's disease," The New England Journal of Medicine, vol. 351, no. 24, pp. 2509-2518, 2004.

[48] D. Burn, M. Emre, I. McKeith et al., "Effects of rivastigmine in patients with and without visual hallucinations in dementia associated with Parkinson's disease," Movement Disorders, vol. 21, no. 11, pp. 1899-1907, 2006.

[49] C. G. Goetz, J. Wuu, L. M. Curgian, and S. Leurgans, "Hallucinations and sleep disorders in PD: six-year prospective longitudinal study," Neurology, vol. 64, no. 1, pp. 81-86, 2005.

[50] E. B. Forsaa, J. P. Larsen, T. Wentzel-Larsen et al., "A 12-year population-based study of psychosis in Parkinson disease," Archives of Neurology, vol. 67, no. 8, pp. 996-1001, 2010.

[51] C. L. Comella, C. M. Tanner, and R. K. Ristanovic, "Polysomnographic sleep measures in Parkinson's disease patients with treatment-induced hallucinations," Annals of Neurology, vol. 34, no. 5, pp. 710-714, 1993.

[52] R. Manni, C. Pacchetti, M. Terzaghi, I. Sartori, F. Mancini, and G. Nappi, "Hallucinations and sleep-wake cycle in PD: a 24-hour continuous polysomnographic study," Neurology, vol. 59, no. 12, pp. 1979-1981, 2002.

[53] T. Nomura, Y. Inoue, H. Mitani, R. Kawahara, M. Miyake, and K. Nakashima, "Visual hallucinations as REM sleep behavior disorders in patients with Parkinson's disease," Movement Disorders, vol. 18, no. 7, pp. 812-817, 2003.

[54] C. B. Saper, T. C. Chou, and T. E. Scammell, "The sleep switch: hypothalamic control of sleep and wakefulness," Trends in Neurosciences, vol. 24, no. 12, pp. 726-731, 2001.

[55] H. H. Fernandez, M. S. Okun, R. L. Rodriguez et al., "Quetiapine improves visual hallucinations in parkinson disease but not through normalization of sleep architecture: results from a double-blind clinical-polysomnography study," International Journal of Neuroscience, vol. 119, no. 12, pp. 2196-2205, 2009.

[56] W. Birkmayer and P. Riederer, "Responsibility of extrastriatal areas for the appearance of psychotic symptoms. (Clinical and biochemical human post mortem findings)," Journal of Neural Transmission, vol. 37, no. 2, pp. 175-182, 1975.

[57] B. Ballanger, A. P. Strafella, T. Van Eimeren et al., "Serotonin 2A receptors and visual hallucinations in Parkinson disease," Archives of Neurology, vol. 67, no. 4, pp. 416-421, 2010.

[58] P. Huot, T. H. Johnston, T. Darr et al., "Increased 5-HT2A receptors in the temporal cortex of Parkinsonian patients with visual hallucinations," Movement Disorders, vol. 25, no. 10, pp. 1399-1408, 2010.

[59] G. Meco and S. Bernardi, "Antidepressant use in treatment of psychosis with comorbid depression in Parkinson's disease," Progress in Neuro-Psychopharmacology and Biological Psychiatry, vol. 31, no. 1, pp. 311-313, 2007.

[60] V. Voon, S. Fox, T. R. Butler, and A. E. Lang, "Antidepressants and psychosis in Parkinson disease: a case series," International Journal of Geriatric Psychiatry, vol. 22, no. 6, pp. 601604, 2007.

[61] V. Voon and A. E. Lang, "Antidepressants in the treatment of psychosis with comorbid depression in Parkinson disease," Clinical Neuropharmacology, vol. 27, no. 2, pp. 90-92, 2004.
[62] C. G. Goetz, C. M. Tanner, and H. L. Klawans, "Bupropion in Parkinson's disease,” Neurology, vol. 34, no. 8, pp. 1092-1094, 1984.

[63] E. C. Lauterbach, "Dopaminergic hallucinosis with fluoxetine in Parkinson's disease," American Journal of Psychiatry, vol. 150, no. 11, p. 1750, 1993.

[64] C. Normann, B. Hesslinger, S. Frauenknecht, M. Berger, and J. Walden, "Psychosis during chronic levodopa therapy triggered by the new antidepressive drug mirtazapine," Pharmacopsychiatry, vol. 30, no. 6, pp. 263-265, 1997.

[65] C. Wlodarek, J. Wuu, and C. G. Goetz, "The effect of SSRI's on hallucinations in Parkinson's disease patients with depression," Annals of Neurology, vol. 60, no. S3, p. S82, 2006.

[66] L. Marsh, J. R. Williams, M. Rocco, S. Grill, C. Munro, and T. M. Dawson, "Psychiatric comorbidities in patients with Parkinson disease and psychosis," Neurology, vol. 63, no. 2, pp. 293-300, 2004.

[67] N. Ibarretxe-Bilbao, B. Ramirez-Ruiz, C. Junque et al., "Differential progression of brain atrophy in Parkinson's disease with and without visual hallucinations," Journal of Neurology, Neurosurgery and Psychiatry, vol. 81, no. 6, pp. 650-657, 2010.

[68] B. Ramírez-Ruiz, M. J. Martí, E. Tolosa et al., "Brain response to complex visual stimuli in Parkinson's patients with hallucinations: a functional magnetic resonance imaging study," Movement Disorders, vol. 23, no. 16, pp. 2335-2343, 2008.

[69] A. M. Meppelink, B. M. De Jong, R. Renken, K. L. Leenders, F. W. Cornelissen, and T. Van Laar, "Impaired visual processing preceding image recognition in Parkinson's disease patients with visual hallucinations," Brain, vol. 132, no. 11, pp. 2980 2993, 2009.

[70] K. Okada, N. Suyama, H. Oguro, S. Yamaguchi, and S. Kobayashi, "Medication-induced hallucination and cerebral blood flow in Parkinson's disease," Journal of Neurology, vol. 246, no. 5, pp. 365-368, 1999.

[71] N. Oishi, F. Udaka, M. Kameyama, N. Sawamoto, K. Hashikawa, and H. Fukuyama, "Regional cerebral blood flow in Parkinson disease with nonpsychotic visual hallucinations," Neurology, vol. 65, no. 11, pp. 1708-1715, 2005.

[72] A. Nagano-Saito, Y. Washimi, Y. Arahata et al., "Visual hallucination in Parkinson's disease with FDG PET," Movement Disorders, vol. 19, no. 7, pp. 801-806, 2004.

[73] M. E. L. Arbouw, J. P. P. van Vugt, T. C. G. Egberts, and H. J. Guchelaar, "Pharmacogenetics of antiparkinsonian drug treatment: a systematic review," Pharmacogenomics, vol. 8, no. 2, pp. 159-176, 2007.

[74] L. Skipper, J. J. Liu, and E. K. Tan, "Polymorphisms in candidate genes: implications for the current treatment of Parkinson's disease," Expert Opinion on Pharmacotherapy, vol. 7, no. 7, pp. 849-855, 2006.

[75] A. J. Makoff, J. M. Graham, M. J. Arranz et al., "Association study of dopamine receptor gene polymorphisms with druginduced hallucinations in patients with idiopathic Parkinson's disease," Pharmacogenetics, vol. 10, no. 1, pp. 43-48, 2000.

[76] C. G. Goetz, P. F. Burke, S. Leurgans et al., "Genetic variation analysis in Parkinson disease patients with and without hallucinations: case-control study," Archives of Neurology, vol. 58, no. 2, pp. 209-213, 2001.

[77] J. Wang, C. Zhao, B. Chen, and Z. L. Liu, "Polymorphisms of dopamine receptor and transporter genes and hallucinations in Parkinson's disease," Neuroscience Letters, vol. 355, no. 3, pp. 193-196, 2004. 
[78] R. L. Oliveri, G. Annesi, M. Zappia et al., "Dopamine $\mathrm{D}$ receptor gene polymorphism and the risk of levodopainduced dyskinesias in PD," Neurology, vol. 53, no. 7, pp. 1425-1430, 1999.

[79] J. Wang, Z. L. Liu, and B. Chen, "Association study of dopamine D2, D3 receptor gene polymorphisms with motor fluctuations in PD," Neurology, vol. 56, no. 12, pp. 1757-1759, 2001.

[80] R. Kaiser, A. Hofer, A. Grapengiesser et al., "L-Dopainduced adverse effects in PD and dopamine transporter gene polymorphism," Neurology, vol. 60, no. 11, pp. 1750-1755, 2003.

[81] J. G. Goldman, C. G. Goetz, E. Berry-Kravis et al., "Genetic polymorphisms of the dopamine transporter gene and hallucinations in Parkinson's disease," Movement Disorders, vol. 19, no. 9, p. S356, 2004.

[82] K. P. Lesch, D. Bengel, A. Heils et al., "Association of anxiety-related traits with a polymorphism in the serotonin transporter gene regulatory region," Science, vol. 274, no. 5292, pp. 1527-1531, 1996.

[83] A. D. Ogilvie, S. Battersby, V. J. Bubb et al., "Polymorphism in serotonin transporter gene associated with susceptibility to major depression," The Lancet, vol. 347, no. 9003, pp. 731733, 1996.

[84] K. P. Lesch, U. Balling, J. Gross et al., "Organization of the human serotonin transporter gene," Journal of Neural Transmission, vol. 95, no. 2, pp. 157-162, 1994.

[85] M. A. Menza, B. Palermo, R. DiPaola, J. I. Sage, and M. H. Ricketts, "Depression and anxiety in Parkinson's disease: possible effect of genetic variation in the serotonin transporter," Journal of Geriatric Psychiatry and Neurology, vol. 12, no. 2, pp. 49-52, 1999.

[86] S. J. McCann, M. E. McManus, A. G. Johnson, G. D. Mellick, D. G. Le Couteur, and S. M. Pond, "The serotonin transporter gene and Parkinson's disease," European Neurology, vol. 44, no. 2, pp. 108-111, 2000.

[87] J. G. Goldman, C. G. Goetz, E. Berry-Kravis et al., "Serotonin transporter gene polymorphisms and hallucinations in Parkinson's disease," Annals of Neurology, vol. 56, no. S8, p. S26, 2004.

[88] L. Kiferle, R. Ceravolo, L. Petrozzi et al., "Visual hallucinations in Parkinson's disease are not influenced by polymorphisms of serotonin 5-HT2A receptor and transporter genes," Neuroscience Letters, vol. 422, no. 3, pp. 228-231, 2007.

[89] J. Wei and G. P. Hemmings, "The CCK-A receptor gene possibly associated with auditory hallucinations in schizophrenia," European Psychiatry, vol. 14, no. 2, pp. 67-70, 1999.

[90] J. N. Crawley, "Cholecystokinin-dopamine interactions," Trends in Pharmacological Sciences, vol. 12, no. 6, pp. 232236, 1991.

[91] C. Fujii, S. Harada, N. Ohkoshi et al., "Association between polymorphism of the cholecystokinin gene and idiopathic Parkinson's disease," Clinical Genetics, vol. 56, no. 5, pp. 394399, 1999.

[92] T. V. O. Hansen, "Cholecystokinin gene transcription: promoter elements, transcription factors and signaling pathways," Peptides, vol. 22, no. 8, pp. 1201-1211, 2001.

[93] J. Wang, Y. M. Si, Z. L. Liu, and L. Yu, "Cholecystokinin, cholecystokinin-A receptor and cholecystokinin-B receptor gene polymorphisms in Parkinson's disease," Pharmacogenetics, vol. 13, no. 6, pp. 365-369, 2003.
[94] J. G. Goldman, C. G. Goetz, E. Berry-Kravis, S. Leurgans, and L. Zhou, "Genetic polymorphisms in Parkinson disease subjects with and without hallucinations: an analysis of the cholecystokinin system," Archives of Neurology, vol. 61, no. 8, pp. 1280-1284, 2004.

[95] R. De La Fuente-Fernández, M. A. Núñez, and E. López, “The apolipoprotein E $\varepsilon 4$ allele increases the risk of drug-induced hallucinations in Parkinson's disease," Clinical Neuropharmacology, vol. 22, no. 4, pp. 226-230, 1999.

[96] R. Camicioli, A. Rajput, M. Rajput et al., "Apolipoprotein E $\varepsilon 4$ and catechol-O-methyltransferase alleles in autopsyproven Parkinson's disease: relationship to dementia and hallucinations," Movement Disorders, vol. 20, no. 8, pp. 989994, 2005.

[97] R. E. Featherstone, S. Kapur, and P. J. Fletcher, "The amphetamine-induced sensitized state as a model of schizophrenia," Progress in Neuro-Psychopharmacology and Biological Psychiatry, vol. 31, no. 8, pp. 1556-1571, 2007.

[98] F. Owen, H. F. Baker, and R. M. Ridley, "Effect of chronic amphetamine administration on dopaminergic systems in the vervet brain: relationship to findings in the brains of schizophrenics," Biochemical Society Transactions, vol. 11, no. 1, pp. 68-69, 1983.

[99] R. M. Ridley, H. F. Baker, and F. Owen, "Behavioural and biochemical effects of chronic amphetamine treatment in the vervet monkey," Psychopharmacology, vol. 78, no. 3, pp. 245251, 1982.

[100] S. H. Fox, N. P. Visanji, T. H. Johnston, J. Gomez-Ramirez, V. Voon, and J. M. Brotchie, "Dopamine receptor agonists and levodopa and inducing psychosis-like behavior in the MPTP primate model of Parkinson disease," Archives of Neurology, vol. 63, no. 9, pp. 1343-1344, 2006.

[101] N. P. Visanji, J. Gomez-Ramirez, T. H. Johnston et al., "Pharmacological characterization of psychosis-like behavior in the MPTP-lesioned nonhuman primate model of Parkinson's disease," Movement Disorders, vol. 21, no. 11, pp. 1879-1891, 2006.

[102] J. Song, D. Hanniford, C. Doucette et al., "Development of homogenous high-affinity agonist binding assays for 5-HT2 receptor subtypes," Assay and Drug Development Technologies, vol. 3, no. 6, pp. 649-659, 2005.

[103] K. E. Vanover, A. J. Betz, S. M. Weber et al., "A 5-HT receptor inverse agonist, ACP-103, reduces tremor in a rat model and levodopa-induced dyskinesias in a monkey model," Pharmacology Biochemistry and Behavior, vol. 90, no. 4, pp. 540-544, 2008.

[104] S. A. Factor and J. H. Friedman, "The emerging role of clozapine in the treatment of improvement disorders," Movement Disorders, vol. 12, no. 4, pp. 483-496, 1997.

[105] J. H. Friedman, "Parkinson's disease psychosis 2010: a review article," Parkinsonism and Related Disorders, vol. 16, no. 9, pp. 553-560, 2010.

[106] H. Y. Meltzer, J. I. Koenig, J. F. Nash, and G. A. Gudelsky, "Melperone and clozapine: neuroendocrine effects of atypical neuroleptic drugs," Acta Psychiatrica Scandinavica, Supplement, vol. 80, no. 352, pp. 24-29, 1989.

[107] H. Y. Meltzer, S. Matsubara, and J.-C. Lee, "The ratios of serotonin2 and dopamine2 affinities differentiate atypical and typical antipsychotic drugs," Psychopharmacology Bulletin, vol. 25 , no. 3, pp. 390-392, 1989.

[108] L. Barbato, A. Monge, F. Stocchi, and G. Nordera, "Melperone in the treatment of iatrogenic psychosis in Parkinson's disease," Functional Neurology, vol. 11, no. 4, pp. 201-207, 1996. 
[109] A. Abbas and B. L. Roth, "Pimavanserin tartrate: a 5-HT2A inverse agonist with potential for treating various neuropsychiatric disorders," Expert Opinion on Pharmacotherapy, vol. 9, no. 18, pp. 3251-3259, 2008.

[110] C. Roberts, "ACP-103, a 5-HT receptor inverse agonist," Current Opinion in Investigational Drugs, vol. 7, no. 7, pp. 653-660, 2006.

[111] H. Y. Meltzer, R. Mills, S. Revell et al., "Pimavanserin, a serotonin 2A receptor inverse agonist, for the treatment of Parkinson's disease psychosis," Neuropsychopharmacology, vol. 35, no. 4, pp. 881-892, 2010.

[112] R. B. Friedman, J. H. Mills, R. Mills et al., "A multicenter, placebo controlled, double blind trial to examine the safety and efficacy of pimavanserin in the treatment of psychosis in Parkinson's disease," Neurology, vol. 74, no. S2, p. A299, 2010.

[113] B. R. Teegarden, H. Al Shamma, and Y. Xiong, "5-HT2A inverse-agonists for the treatment of insomnia," Current Topics in Medicinal Chemistry, vol. 8, no. 11, pp. 969-976, 2008.

[114] J. H. Friedman, "Low-dose clozapine for the treatment of drug-induced psychosis in Parkinson's disease. The Parkinson Study Group," The New England Journal of Medicine, vol. 340, no. 10, pp. 757-763, 1999.

[115] L. Morgante, A. Epifanio, E. Spina et al., "Quetiapine versus clozapine: a preliminary report of comparative effects on dopaminergic psychosis in patients with Parkinson's disease," Neurological Sciences, vol. 23, supplement 2, pp. S89-S90, 2002.

[116] A. Breier, V. K. Sutton, P. D. Feldman et al., "Olanzapine in the treatment of dopamimetic-induced psychosis in patients with Parkinson's disease," Biological Psychiatry, vol. 52, no. 5, pp. 438-445, 2002.

[117] W. G. Ondo, J. K. Levy, K. D. Vuong, C. Hunter, and J. Jankovic, "Olanzapine treatment for dopaminergic-induced hallucinations," Movement Disorders, vol. 17, no. 5, pp. 10311035, 2002.

[118] J. M. Miyasaki, K. Shannon, V. Voon et al., "Practice parameter: evaluation and treatment of depression, psychosis, and dementia in Parkinson disease (an evidencebased review): report of the Quality Standards Subcommittee of the American Academy of Neurology," Neurology, vol. 66, no. 7, pp. 996-1002, 2006.

[119] R. Kurlan, J. Cummings, R. Raman, and L. Thal, "Quetiapine for agitation or psychosis in patients with dementia and parkinsonism," Neurology, vol. 68, no. 17, pp. 1356-1363, 2007.

[120] W. G. Ondo, R. Tintner, K. D. Voung, D. Lai, and G. Ringholz, "Double-blind, placebo-controlled, unforced titration parallel trial of quetiapine for dopaminergic-induced hallucinations in Parkinson's disease," Movement Disorders, vol. 20, no. 8, pp. 958-963, 2005. 


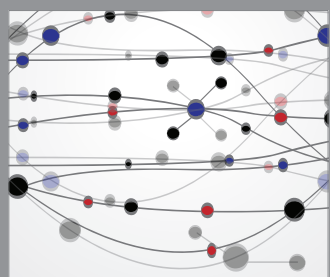

The Scientific World Journal
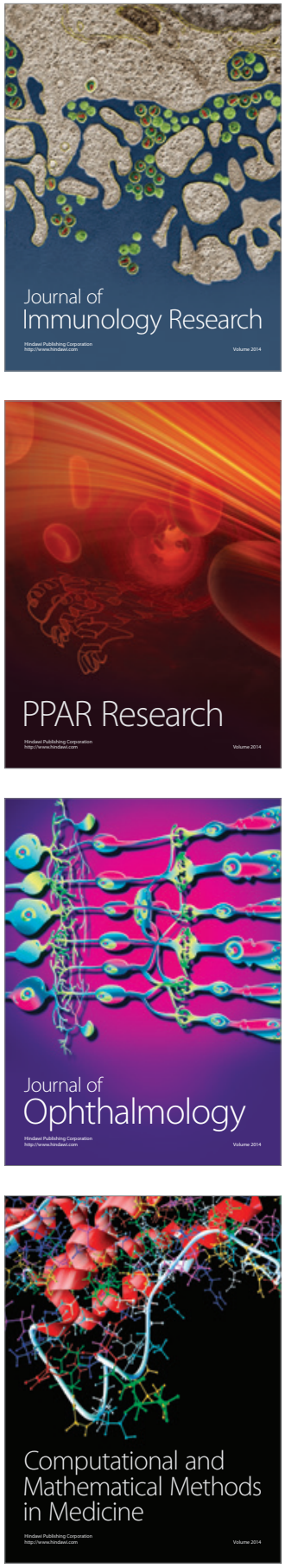

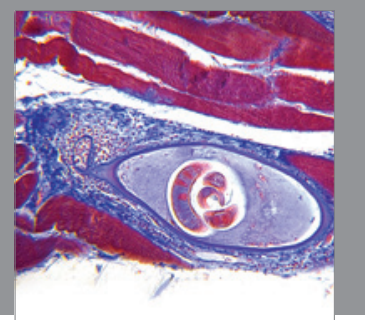

Gastroenterology

Research and Practice
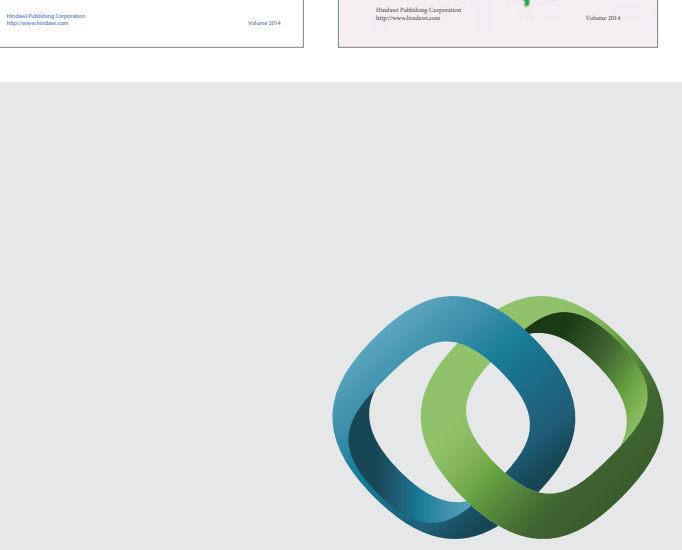

\section{Hindawi}

Submit your manuscripts at

http://www.hindawi.com
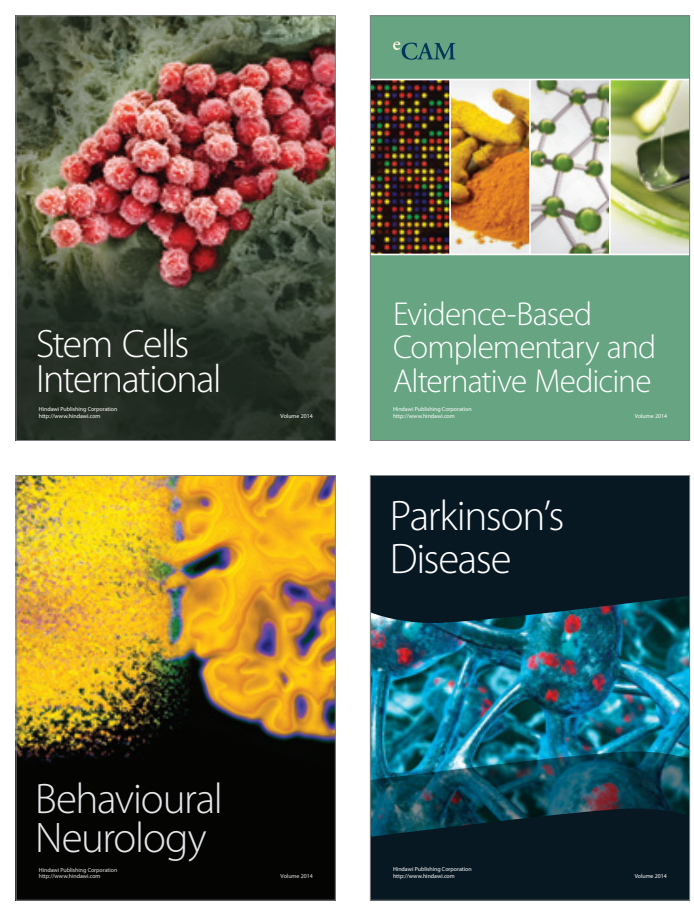

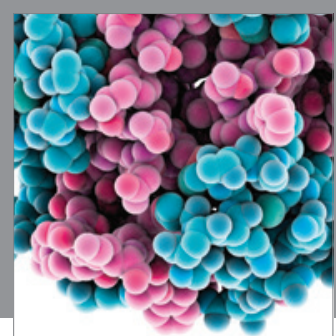

Journal of
Diabetes Research

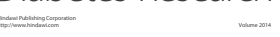

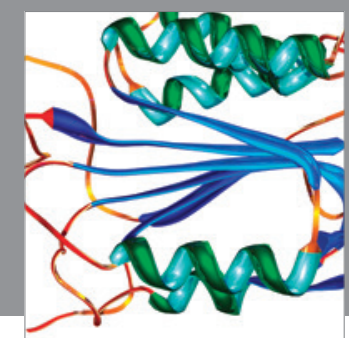

Disease Markers
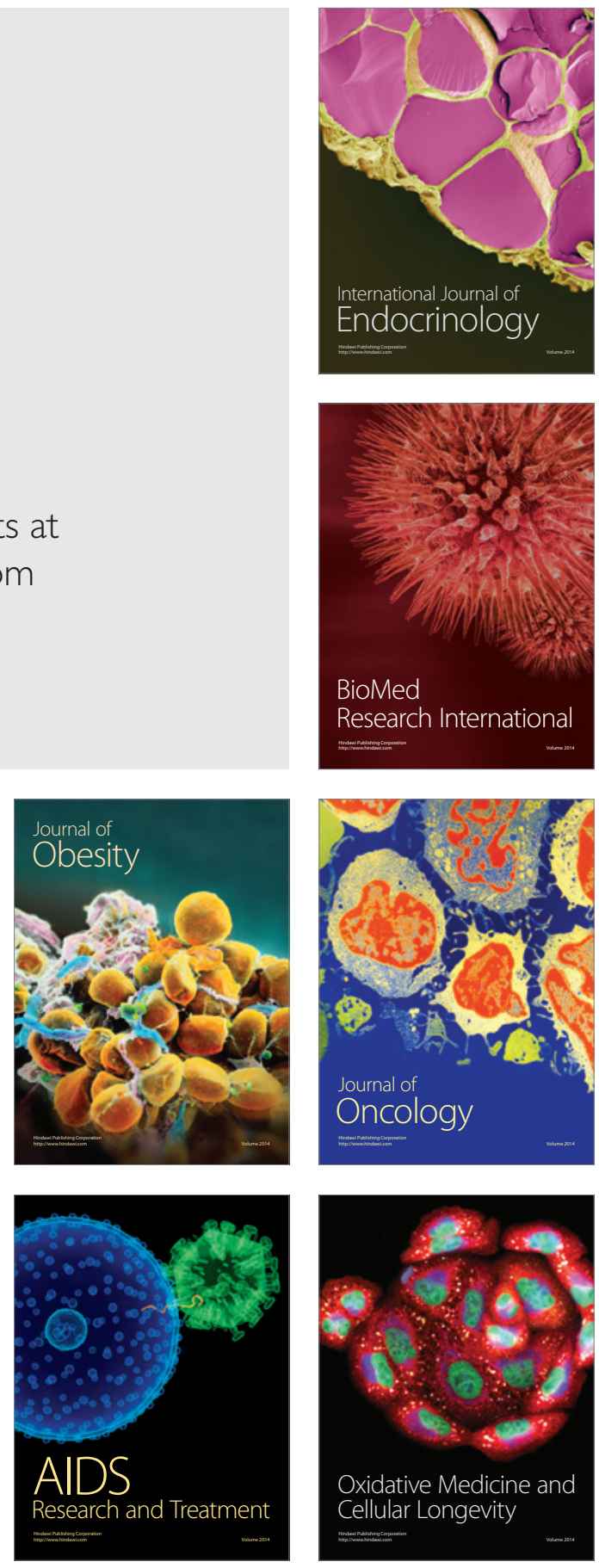